lässt, kann ein methodisch standardisierter und klinisch validierter Multigentest für die Entscheidung herangezogen werden. Betont wird, dass hinsichtlich des Nutzens der Multigentests noch weiterer Forschungsbedarf besteht.

\section{BET: Wächerlymphknoten entfernen oder nicht?}

Ist die axilläre Lymphknotenentfernung bald überflüssig? Dieses Thema wird laut Toralf Reimer, Rostock, künftig bei Mammakarzinompatientinnen mit unauffälligem Tastbefund und normalem Ultraschallbefund der Achselhöhle vor einer geplanten brusterhaltenden Therapie (BET) diskutiert werden. Derzeit werden in dieser Situation die Sentinellymphknoten entfernt - mit dem Risiko eines Lymphödems.

Zur Klärung beitragen soll die Studie INSEMA(Intergroup-Sentinel-Mamma). In ihr wird untersucht, ob der Verzicht auf diese Maßnahme onkologisch sicher ist und Komplikationen verringert, aber auch ob bei Befall des Wächterlymphknotens mit Makrometastasen eine komplette Axilla-Operation zwingend ist. Bislang sind 2.000 Patientinnen mit frühem Brustkrebs und geplanter BET in die Studie aufgenommen, 6.000 Patientinnen sollen es werden. Spannende Ergebnisse lieferte jetzt eine erste Analyse nach fast 2.000 Operationen. Im Kontrollarm, der standardmäßigen Durchführung der Sentinellymphknotenbiopsie, waren $83 \%$ der Frauen nodal-negativ, bei 2,8\% fanden sich nicht relevante Mikrometastasen. Nodal-positiv waren nur $14 \%$. „Erwartet wurden 25 bis $30 \%$ “, so Studienleiter Reimer.

\section{Chemotherapiestrategien zur Eskalation und Deeskalation}

Zielgerichtete Therapiestrategien gegen den Tumor dominieren derzeit die Entwicklung in der onkologischen Behandlung. Doch auch die Chemotherapie sei im Wandel, wie Cornelia Liedtke, Lübeck, erklärte. Im Fokus steht die Senkung unnötiger Toxizitäten - ohne Verringerung der Effektivität. Liedtke hat dabei zwei grundsätzliche Entwicklungen beobachtet:

_ die Vermeidung von Über- und Untertherapie durch treffsichere Indikationsstellung,

\title{
CESM ist Ultraschall und Mammografie überlegen
}

Karzinome in einem möglichst frühen Stadium zu entdecken: Das ist die Anforderung an bildgebende Verfahren in der Mammadiagnostik. Besonders in der dichten Brust ist die Sensitivität der Magnetresonanztomografie (MRT) der Mammografie überlegen. Auch die Kontrastmittelunterstützte Spektralmammografie (CESM) ist aussagefähiger, wie Eva Maria Fallenberg, Berlin, anhand eigener Daten erläuterte [Fallenberg EM et al. Breast Cancer Res Treat. 2014;146(2):371-81]. Danach betrug die Sensitivität der Mammografie bei prämenopausalen Frauen 69,6\%, die der CESM 91,1\%. In der Postmenopause wurde eine Sensitivität von 84,3 versus $98,4 \%$ erreicht. Besonders profitierten auch hier Frauen mit einer dichten Brust (Kategorie 3 und 4): Die Mammografie erreichte eine Sensitivität von $71,6 \%$, die CESM von 93,3\%. Bei niedrigerer Dichte (Kategorie 1 und 2) lagen die Werte bei 85,8 und $96,5 \%$. Die Kombination der beiden Verfahren brachte keinen zusätzlichen Nutzen gegenüber der CESM allein. Auch im Vergleich mit dem Ultraschall scheint laut Fallenberg die CESM überlegen, mit einer Sensitivität von 78 versus $66 \%$ [Perez-Fernandez CM et al. Europäischer Radiologenkongress. 2015;Abstr B-0436]. Beate Fessler

Bericht von der 37. Jahrestagung der Deutschen Gesellschaft für Senologie vom 29. Juni bis 1. Juli 2017 in Berlin

_Deeskalation oder Eskalation, je nach Situation, durch Optimierung des Chemotherapieregimes.

Für Patientinnen im palliativen Stadium bedeute dies vor allem gut verträgliche Monotherapien mit dem Ziel, die Lebensqualität zu erhalten, sagte Liedtke. Es gelte, die individuell geeignetste Substanz zu wählen. Anders bei Frauen mit heilbarem Brustkrebs: Sie erhalten meist Kombinationschemotherapien mit mehreren Substanzen, die sequenziell und/ oder simultan angewendet werden. Ist eine Therapieeskalation erforderlich, ist eine Möglichkeit die Hinzunahme zusätzlicher Zytostatika, um die Effektivität zu verbessern. Als Beispiel nannte Liedtke das triplenegative Mammakarzinom (TNBC), bei dem standardisierte Taxan-Anthrazyklin-Chemotherapien durch Carboplatin ergänzt werden.

\section{Zusätzliche zielgerichtete Therapien}

Auch zusätzliche zielgerichtete Therapien sind eine Option, etwa eine duale HER2-Blockade beim HER2-positiven Mammakarzinom. In Subgruppen werden derzeit "postneoadjuvante“ Therapien bei Frauen mit Resttumor trotz präoperativer Chemotherapie untersucht. Zur Therapie-Deeskalation können toxische Komponenten der Standardtherapie ausgetauscht werden. Als Beispiel nannte Liedtke erneut das TNBC: „Erste Studiendaten legen nahe, dass ein Einsatz der Anthrazykline durch das weniger toxische Carboplatin möglich sein könnte.“

\section{PMBC: Primarius entfernen?}

Sollte beim primär metastasierten Mammakarzinom (PMBC) der Primarius belassen oder entfernt werden? Diese Frage stellte sich Jana Barinoff, Berlin. Die Datenlage dazu ist kontrovers. In einer aktuellen Studie [ClinicalTrials.gov: NCT00557986] war das Überleben innerhalb der ersten drei Jahre unabhängig davon, ob der Primarius entfernt wurde oder nicht. Nach fünf Jahren war der Unterschied dagegen signifikant (41,6 vs. $24,4 \%)$. Insbesondere Patientinnen mit ER-positiven, HER2-negativen Tumoren, solitärer Knochenmetastase und einem Alter unter 55 Jahren hatten einen Überlebensvorteil nach initialer Operation.

Letztlich ließen sich beide Konzepte verfolgen, so Barinoff. Wichtig sei eine individuelle Entscheidung. Auch die Arbeitsgemeinschaft Gynäkologische Onkologie (AGO) sieht die Operation des Primärtumors bei alleiniger ossärer Metastasierung als Einzelfallentscheidung $(+/-)$, bei viszeralen Metastasen ist die Bewertung negativ (-). Wenn die Patientin ihren Tumor „loswerden will“, so Jens Huober, Ulm, „würde ich mitgehen“. Es gehe schließlich um die Lebensqualität der Patientin. Dennoch müsse der Primarius immer im Auge behalten werden, so Huober. Beate Fessler

Bericht von der 37. Jahrestagung der Deutschen Gesellschaft für Senologie vom 29. Juni bis 1. Juli 2017 in Berlin 